\title{
ADAPTIVE VIDEO DENOISING USING BLOCK MATCHING 3-D FILTERING
}

\author{
Guangyi Chen ${ }^{a, b}$, Tien D. Bui ${ }^{a}$, Adam Krzyzak ${ }^{a}$ and Stéphane Coulombe ${ }^{b}$ \\ ${ }^{a}$ Department of Computer Science and Software Engineering, Concordia University, Montreal, Quebec, Canada \\ H3G $1 \mathrm{M} 8$. \\ Email: \{guang_c, bui, krzyzak\}@cse.concordia.ca \\ ${ }^{\mathrm{b}}$ Department of Software and IT Engineering, École de technologie supérieure, 1100, rue Notre-Dame Ouest, \\ Montreal, Quebec, Canada H3C 1K3 \\ Email: \{Guangyi.Chen, Stephane.Coulombe\}@etsmtl.ca
}

\begin{abstract}
Reducing noise in a video sequence is of vital importance in many applications. Despite the fact that many good video denoising methods were proposed in recent years, there is still a need to further improve the existing video denoising methods. Video block matching 3-D filtering (VBM3D), which was developed by Dabov et al. in 2007, is one of the recent state-of-the-art video denoising methods of interest. In the VBM3D, it is assumed that the noise standard deviation for the whole video sequence is known in advance. This is an important drawback of this method. In this paper, we propose a new method to reduce the noise in a video frame adaptively. If the noise standard deviation of the video frame is less than a threshold $\tau_{0}$, then we do not apply any denoising method on it. If, on the other hand, the noise standard deviation is greater than $\tau_{0}$, and there are $\mathrm{k}>0$ video frames with noise standard deviation $\sigma_{n}(j)$ satisfying $\left|\sigma_{n}(j)-\sigma_{n}(i)\right|<\varepsilon$ for $j \in[i+1, \ldots, i+k]$, then we apply VBM3D on the $\mathrm{k}$ video frames in order to reduce the noise in these video frames. If the next video frame does not satisfy $\left|\sigma_{n}(i+1)-\sigma_{n}(i)\right|<\varepsilon$, then we apply the 2-D block matching 3-D filtering (BM3D) method to denoise it. We conducted some experiments to reduce the noise in several video sequences, and we found out that our proposed method outperforms VBM3D for denoising video sequences with different noise levels.
\end{abstract}

Index Terms - Video denoising, block matching 3-D filtering (BM3D), video block matching 3-D filtering (VBM3D), hard thresholding, Wiener filter.

\section{INTRODUCTION}

Video denoising has been a very hot research topic for many years. We briefly review the most competitive video denoising algorithms here. Dabov et al. [1] proposed a video block matching 3-D filtering (VBM3D) for reducing noise in a video sequence. This algorithm is based on their 2-D block matching 3-D filtering (BM3D) [2] for image denoising. Both the VBM3D and BM3D algorithms have achieved state-of-the-art performance for reducing noise in a video sequence and a noisy image. Luisier et al. [3] studied the SURE-LET approach for orthonormal wavelet-domain video denoising. Varghese and Wang [4] developed a video denoising algorithm based on a spatiotemporal Gaussian scale mixture model. However, this method is very slow due to its high computational complexity. Zlokolica et al. [5] proposed a wavelet-domain video denoising algorithm based on reliability measures. Balster et al. [6] combined spatial and temporal domain wavelet shrinkage algorithm for video denoising. Rahman et al. [7] proposed a video denoising algorithm by using inter-frame statistical modeling of wavelet coefficients. Protter and Elad [8] studied image sequence denoising via sparse and redundant representations. Examples of the current developments in video denoising also include the wavelet based techniques [9], [10]. These algorithms utilize both the sparsity and the statistical properties of a multiresolution representation as well as the inherent correlations between frames in temporal dimension.

In this paper, we propose a new algorithm for reducing noise in a video sequence. Our algorithm is based on the VBM3D and BM3D algorithms. It is well known that the VBM3D algorithm assumes the common noise standard deviation of the whole video sequence to be known in advance. This is a major drawback of the VBM3D algorithm. We suggest denoising a video sequence adaptively here. If the noise standard deviation of a video frame is greater than a threshold $\tau_{0}$ and the next frame does not satisfy $\left|\sigma_{n}(i+1)-\sigma_{n}(i)\right|<\varepsilon$, then we call the BM3D algorithm to reduce the noise in this video frame. If, on the other hand, 
there are $\mathrm{k}>0$ video frames satisfying $\left|\sigma_{n}(j)-\sigma_{n}(i)\right|<\varepsilon$ for $j \in[i+1, \ldots, i+k]$, then we call the VBM3D algorithm to denoise this sub-video sequence. In this way, we can achieve improved results for video denoising. We conduct a number of experiments for different video sequences and different noise patterns. Our experiments show that our proposed denoising algorithm outperforms the VBM3D algorithm significantly.

\section{PROPOSED METHOD}

In the BM3D algorithm, Dabov et al. [2] proposed an image denoising method based on an enhanced sparse representation in the transform-domain. The enhancement of the sparsity was achieved by grouping similar 2-D image fragments into 3-D data arrays, called "groups". These 3-D groups were achieved by using three successive steps: 3-D transformation of 3-D groups, shrinkage of transformed spectrum, and inverse 3-D transformation. The result is a 3$\mathrm{D}$ estimate that consists of the jointly filtered grouped image blocks. By attenuating the noise, the collaborative filtering reveals even the finest details shared by grouped blocks and at the same time it preserves the essential unique features of each individual block. The filtered blocks are then returned to their original positions. Significant improvement can be obtained by a specially developed collaborative Wiener filtering. Experimental results demonstrated that their proposed method achieved the best denoising performance in terms of both peak signal-to-noise ratio (PSNR) and subjective visual quality.

In recent years, a few state-of-the-art video denoising algorithms were developed. This includes the VBM3D algorithm, which achieves excellent denoising results when the noise standard deviation for the whole video sequence is known before-hand. However, this information is not available in practice. In this paper, we propose to perform the 2-D image denoising method BM3D or the 3-D video denoising algorithm VBM3D adaptively. Our adaptive video denoising strategy is as follows. If the next video frame does not satisfy $\left|\sigma_{n}(i+1)-\sigma_{n}(i)\right|<\varepsilon$, then we apply the BM3D algorithm on it. If, on the other hand, the next $\mathrm{k}>0$ frames satisfy $\left|\sigma_{n}(j)-\sigma_{n}(i)\right|<\varepsilon$ for $j \in[i+1, \ldots, i+k]$, then we apply the VBM3D to this sub-video sequence. Even though the noise standard deviation of the whole video sequence is not known, we can estimate $\sigma_{n}(i)$ of a video frame $f_{i}$ from the finest scale wavelet coefficients ( $\mathrm{HH} 1$ subband) as follows [11]:

$$
\sigma_{n}(i)=\frac{\operatorname{median}\left(\left|y_{j}\right|\right)}{0.6745}, y_{j} \in \text { subband } H H_{1}
$$

We propose the following video denoising algorithm, which can be summarized as follows.

1. Initialize $\mathrm{i}=1$ (first frame).

2. Estimate the noise standard deviation $\sigma_{n}(i)$ of video frame i (denoted $f_{i}$ ).

3. If the noise standard deviation of $f_{i}$ is less than a small threshold $\tau_{0}$, then we do not denoise it. Go to 6 .

4. If the noise standard deviation of $f_{i}$ is greater than $\tau_{0}$ and the next frame does not satisfy $\left|\sigma_{n}(i+1)-\sigma_{n}(i)\right|<\varepsilon$, then apply the 2-D denoising method BM3D to $f_{i}$. Go to 6 .

5. If the next $\mathrm{k}>0$ frames $f_{j}, j \in[i+1, \ldots, i+k]$, satisfy $\left|\sigma_{n}(j)-\sigma_{n}(i)\right|<\varepsilon$, then we apply the VBM3D algorithm to this sub-video sequence.

6. Set $\mathrm{i}=\mathrm{i}+1$. If $i \leq N$ then go to 2 .

We conducted a number of experiments to reduce noise in different video sequences for different noise levels. Our experimental results show that the proposed method in this paper outperforms the VBM3D algorithm significantly. This indicates that our proposed algorithm can perform well in processing noisy video sequences

\section{EXPERIMENTAL RESULTS}

In this section, we perform a series of experiments to test if our proposed video denoising method is better than the VBM3D algorithm, which is one of the few state-of-the-art video denoising algorithms published in the literature. We test our algorithm for different noise standard deviation according to the following three cases, where $\mathrm{i}$ is the frame number.

$$
\begin{aligned}
& \text { Case 1: } \sigma_{n}(\mathrm{i})=\bmod (\mathrm{i}, 25)+1 \\
& \text { Case 2: } \sigma_{n}(\mathrm{i})=2+\text { floor }(\mathrm{i} / 25) * 20 \\
& \text { Case 3: } \sigma_{n}(\mathrm{i})=1+25 *|\operatorname{randn}(1,1)|
\end{aligned}
$$

We only consider Gaussian white noise in this paper. For other types of noise, we leave them as future work. We choose $\tau_{0}=0.1$ and $\varepsilon=0.1$ in our experiments. Since the common noise standard deviation needed by VBM3D is not known, we use the mean of $\sigma_{n}$ (i) as defined in eq. (1) to approximate it in this paper. The peak signal to noise ratio (PSNR) is used as a measure for the quality of a video 
denoising method. We add the MSE for each frame, compute the mean, and then compute the PSNR based on it. We have tested the following video sequences in this section: Camera man $(288 \times 352 \times 50)$, Bus $(288 \times 352 \times 150)$, Coast guard (144x176x300), Bicycle (576x720x30), Flower (240x352x150), Foreman $(288 \times 352 \times 300)$, Miss America $(288 \times 360 \times 150)$, and Tennis $(240 \times 352 \times 150)$. These video sequences are standard testing video sequences, which can be downloaded from the web site: http://www.cs.tut.fi/ foi/GCF-BM3D. We have added Gaussian white noise to these video sequences in order to generate noisy video sequences. The noise standard deviation of a video frame fi is given as $\sigma_{n}$ (i) for the three cases given above. Figs. 1-3 show the noise standard deviations of the three noise cases given above for a video sequence with 150 frames, respectively. These three cases of noise standard deviation cover abrupt changes, piece-wise constant, and random changes as shown in the figures. Table 1 shows the experimental results in terms of PSNR for different video sequences. From this table it can be seen that our proposed method in this paper is significantly better than the VBM3D method for all eight tested video sequences. It should be mentioned that the difference in performance between VBM3D and the proposed method depends on the variation of the noise with time.

Table 1. The PSNR values of the proposed method and the VBM3D algorithm for different video sequences and different noise patterns.

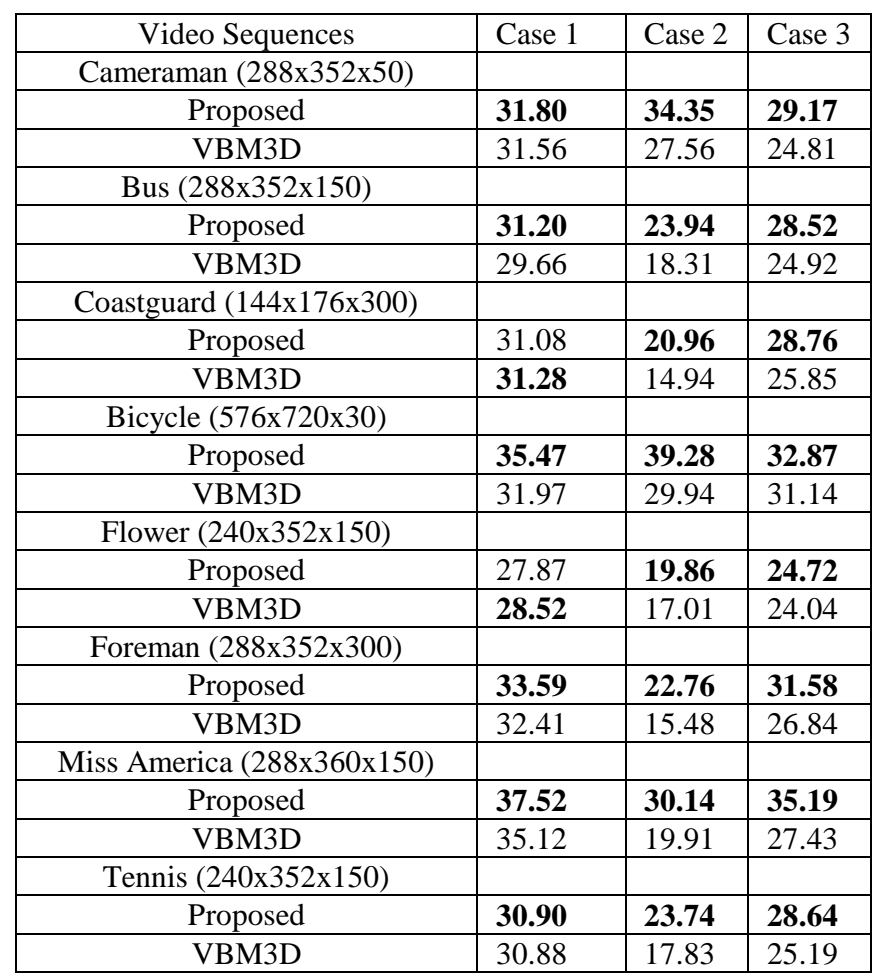

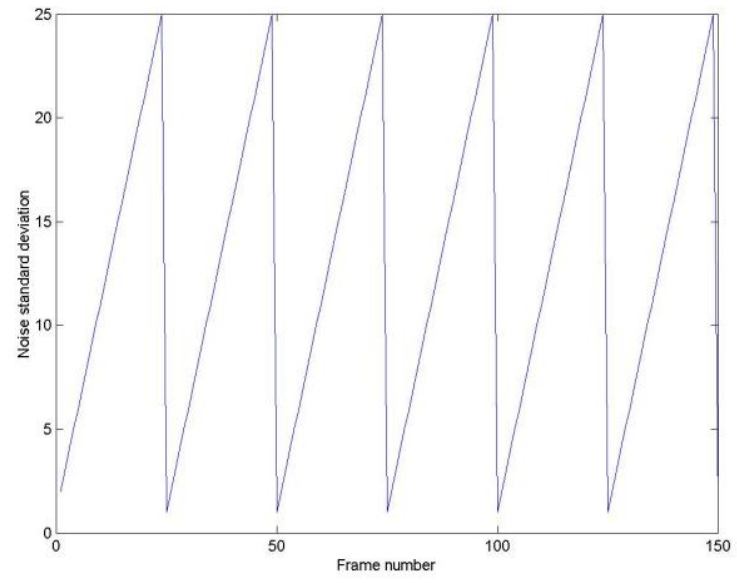

Fig. 1. The noise standard deviation of Case 1.

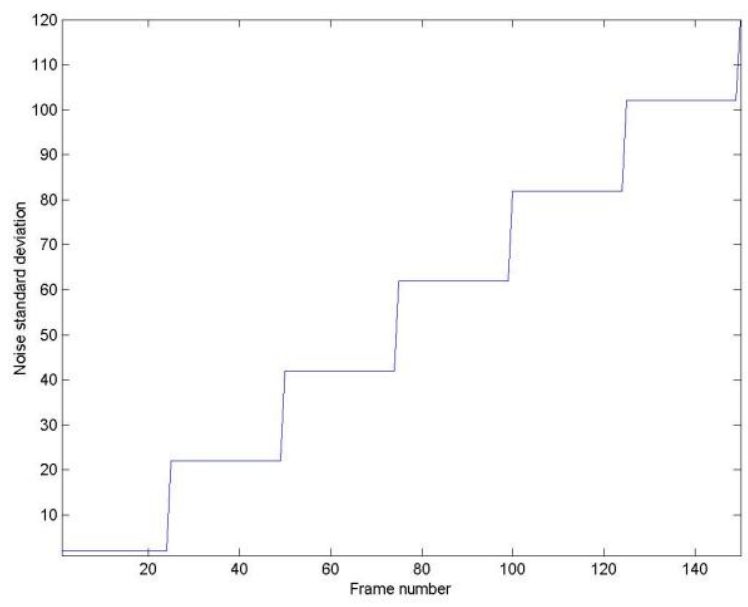

Fig. 2. The noise standard deviation of Case 2.

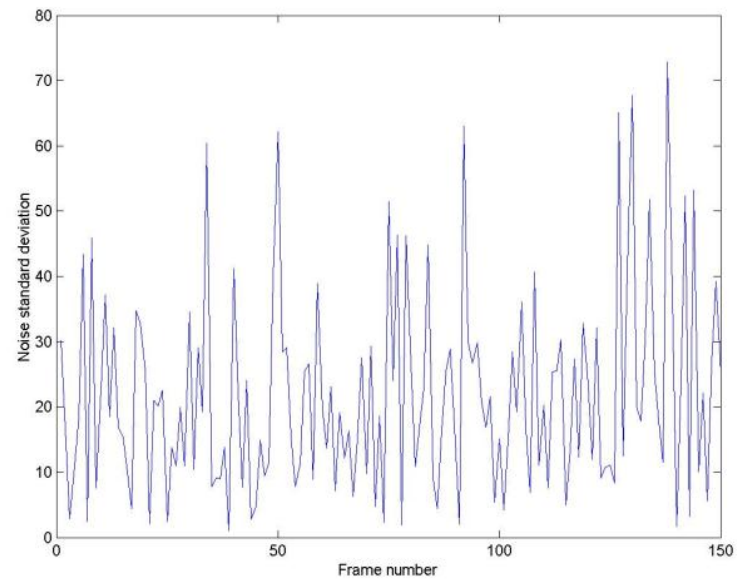

Fig. 3. The noise standard deviation of Case 3. 


\section{CONCLUSION}

Video sequences acquired are often contaminated by noise, and reducing noise in video signals (or video denoising) is highly desirable. This is because video denoising can enhance perceived image quality, facilitate transmission bandwidth reduction, and improve the accuracy of feature extraction, object detection, motion tracking and pattern classification.

In this paper, a new adaptive video denoising algorithm has been proposed by using block matching 3-D filtering. It is well known that the VBM3D algorithm for video denoising assumes that the noise standard deviation is available and constant for the whole video sequence. However, this is not true in practice. We propose to use the noise standard deviation of the current video frame to decide whether we choose the BM3D to denoise this video frame or the VBM3D to denoise the sub-video sequence with a common noise standard deviation. Experimental results conducted in this paper show that the proposed video denoising algorithm outperforms the VBM3D algorithm significantly when the noise standard deviation changes dynamically for different video frames.

Further investigation is needed in order to improve the existing video denoising methods published in the literature. We believe that compressive sensing could be applicable for reducing noise in noisy video sequences. We will consider using minimum noise fraction (MNF) to decorrelate the useful features from the noise, and then denoise those noisy frames. An inverse MNF transform will obtain the denoised video sequence. It is also interesting to compare our proposed video denoising method with a method that denoises every video frame separately by using BM3D. The above discussion in this paper only applies to Gaussian white noise. Other types of noise will be studied in the near future.

\section{ACKNOWLEDGMENT}

This work was supported by the research grants from the Natural Science and Engineering Research Council of Canada (NSERC).

\section{REFERENCES}

[1] K. Dabov, A. Foi and K. Egiazarian, "Video denoising by sparse 3D transform-domain collaborative filtering," Proceedings of the 15th European Signal Processing Conference, EUSIPCO 2007, Poznan, Poland, Sept. 2007.
[2] K. Dabov, A. Foi, V. Katkovnik and K. Egiazarian, "Image denoising by sparse 3D transform-domain collaborative filtering," IEEE Transactions on Image Processing, vol. 16, no. 8, pp. 2080-2095, Aug. 2007.

[3] F. Luisier, T. Blu and M. Unser, "SURE-LET for orthonormal wavelet-domain video denoising," IEEE Transactions on Circuits and Systems for Video Technology, vol. 20, no. 6, pp. 913-919, 2010.

[4] G. Varghese and Z. Wang, "Video denoising based on a spatiotemporal Gaussian scale mixture model," IEEE Transactions on Circuit and Systems for Video Technology, vol. 20, no. 7, pp. 1032-1040, Jul. 2010.

[5] V. Zlokolica, A. Pizurica and W. Philips, "Waveletdomain video denoising based on reliability measures," IEEE Transactions on Circuits Systems for Video Technology, vol. 16, no. 8, pp. 993-1007, Aug. 2006.

[6] E. J. Balster, Y. F. Zheng and R. L. Ewing, "Combined spatial and temporal domain wavelet shrinkage algorithm for video denoising," IEEE Transactions on Circuits Systems for Video Technology, vol. 16, no. 2, pp. 220-230, Feb. 2006.

[7] S. M. M. Rahman, F. M. Omair Ahmad and M. N. S. Swamy, "Video denoising based on inter-frame statistical modeling of wavelet coefficients," IEEE Transactions on Circuits Systems for Video Technology, vol. 17, no. 2, pp. 187-198, Feb. 2007.

[8] M. Protter and M. Elad, "Image sequence denoising via sparse and redundant representations," IEEE Transactions on Image Processing, vol. 18, no. 1, pp. 27-35, Jan. 2009.

[9] F. Jin, P. Fieguth, and L. Winger, "Wavelet video denoising with regularized multiresolution motion estimation," EURASIP Journal on Applied Signal Processing, vol. 2006, pp. Article ID 72705, 11 pages, 2006.

[10] I. Selesnick and K. Li, "Video denoising using 2D and 3D dual-tree complex wavelet transforms," Proceedings of Wavelet Application in Signal and Image Processing X, SPIE, San Diego, USA, Aug. 2003.

[11] D. L. Donoho and I. M. Johnstone, "Ideal spatial adaptation by wavelet shrinkage," Biometrika, vol. 81, no. 3, pp. 425-455, 1994. 